\title{
New Standards for the Validation of EMC Test Sites particularly above $1 \mathrm{GHz}$
}

\author{
S. Battermann ${ }^{1}$, F. W. Trautnitz ${ }^{2}$, and H. Garbe ${ }^{1}$ \\ ${ }^{1}$ Institut für Grundlagen der Elektrotechnik und Messtechnik, University of Hannover, Appelstrasse 9A, 30167 Hannover, \\ Germany \\ ${ }^{2}$ Albatross Projects GmbH, Daimlerstraße 17, 89564 Nattheim, Germany
}

\begin{abstract}
Standards for the validation of alternative test sites with conducting groundplane exist for the frequency range $30-1000 \mathrm{MHz}$ since the end of the eighties. Recently the procedure for fully anechoic rooms (FAR) has been included in CISPR 16 after more than 10 years intensive discussion in standards committees (CENELEC, 2002; CISPR, 2004). But there are no standards available for the validation of alternative test sites above $1 \mathrm{GHz}$. The responsible working group (WG1) in CISPR/A has drawn up the 7th common draft (CD). A CDV will be published in spring 2005. The German standards committee VDE AK 767.4.1 participates in the drafting of the standard. All suggested measurement procedures proposed in the last CDs have been investigated by measurements and theoretical analysis. This contribution describes the basic ideas and problems of the validation procedure of the test site. Furthermore measurement results and numerical calculations will be presented especially for the use of omni-directional antennas.
\end{abstract}

\section{Introduction}

\subsection{FAR ideal and real - differences}

In the ideal case the FAR has no reflections and measurements can be performed under free-space conditions without a ground plane. But in real case a lot of components have to be integrated in the fully anechoic room such as turntable, double floor, masts, cameras, lightning, airing openings, cabling. And last but not least the used absorbers in the room have to satisfy the relevant standards with low reflectivity for the entire frequency range and all incident angles (Holloway et al., 1997).

As can be seen in Fig. 1 the resulting field at the receiving antenna (2) is a superposition of the desired signal from the direct path $s_{\text {direct }}$ and unwanted reflected signals like $s_{\text {refl.1 }}$

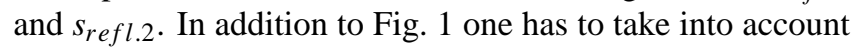

Correspondence to: S. Battermann

(batterma@geml.uni-hannover.de) the 3-D case with reflections from all 6 walls. These reflections cause an interference pattern at the receiving antenna that influences the measurements. Therefore possible reflections have to be measured and quantified during the validation procedure.

\subsection{Radiation pattern of an equipment under test}

Figure 2 shows the radiation pattern of a simple two-slot radiator at $5 \mathrm{GHz}$ fed with a phase shift (two magnetic dipoles) as exemplary equipment under test (EUT). Significant side lobes are visible which may cause reflections at the chamber walls. Generally most EUTs in the frequency range above $1 \mathrm{GHz}$ have to be dealt as a "large EUT" with their dimensions comparable with the wavelength. This results in a very complex radiation pattern. The validation procedure has to take into account these possible patterns.

It is a consequence that an isotropic source would be the best source to illuminate the whole room and to find all unwanted reflections during the validation procedure. Unfortunately it is a problem to realize this kind of electromagnetic source.

\subsection{Typically used validation methods}

The site qualification in the frequency range from $30-1000 \mathrm{MHz}$ is done by measuring the Normalized Site Attenuation (NSA) in the EUT volume according to the "volume method". Contrary to the NSA measurement CISPR/A proposed a different procedure for the site qualification above $1 \mathrm{GHz}$. Similar to the free space VSWR measurement (IEEE, 1979) the so-called "Site-VSWR" is to be measured (AppelHansen, 1973). During discussion in standards committees over the last years, the main problem was the missing omnidirectional antenna.

\subsubsection{Validation with directional antennas}

The first validations have been carried out with high directional antennas (e.g. logarithmic-periodic or horn antennas). A double-ridged horn antenna covers the frequency range 


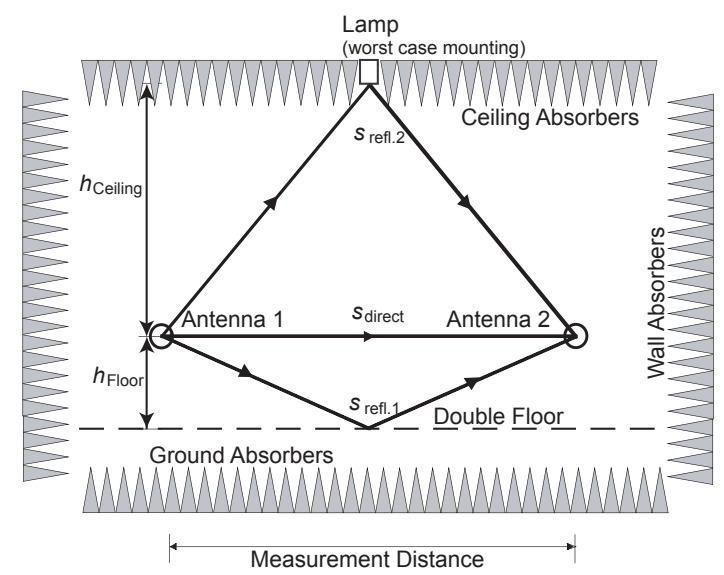

Fig. 1. Superposition of direct and reflected signal.

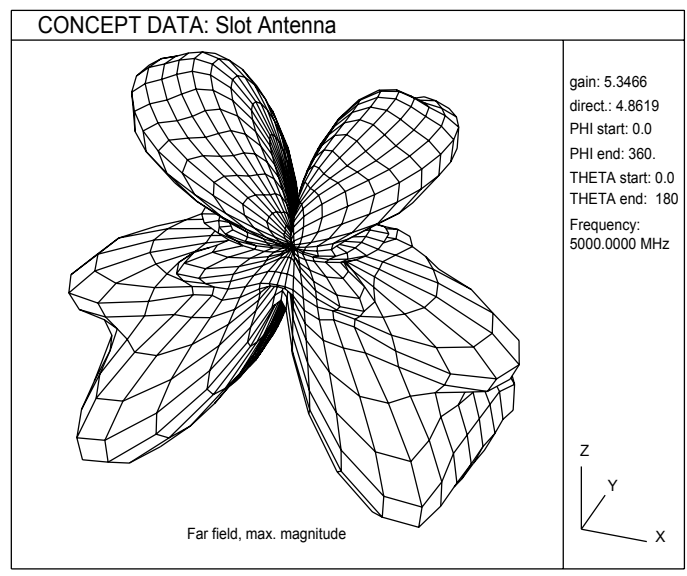

Fig. 2. Radiation pattern of a slot radiator.

from 1-18 GHz, therefore an antenna change can be avoided. But due to the small $3 \mathrm{~dB}$ beam-width of the horn the room characteristics cannot be observed when the antennas are faced to each other. The magnitude of reflected signals by the walls can nearly be neglected (Fig. 3). Therefore this method is inappropriate for room validation, because it will not test the room characteristics.

In a different interesting approach one antenna is fixed and the other antenna will be rotated from $90^{\circ}$ to $270^{\circ}$ to detect reflections from different angles of the back wall (Windler and Camell, 2003). In this case the low front to back ratio of horn antennas for the low frequency range is a problem. Thus it is impossible to separate the reflection by the backside wall and the back lobe of the antenna, which results in a low reproducibility. This is the reason why this method was no longer under consideration.

\subsubsection{Validation with omni-directional antennas}

Monopole antennas (Fig. 4 right) show a strong frequency dependent radiation pattern. Figures 5 and 6 show the vertical radiation pattern of a conical monopole antenna (discone)
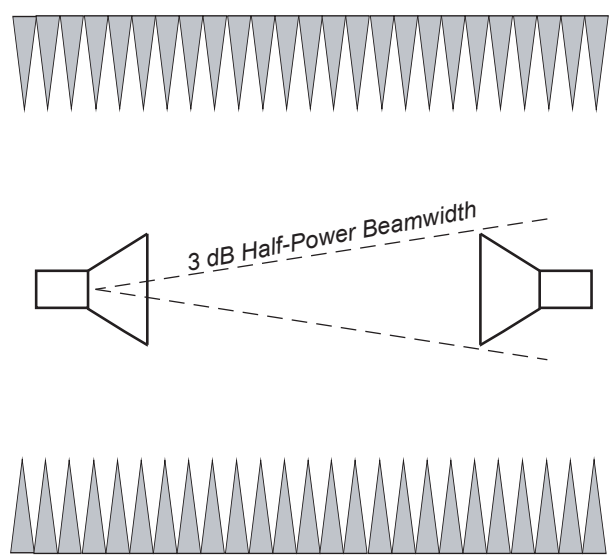

Fig. 3. Insufficient validation with directional antennas.
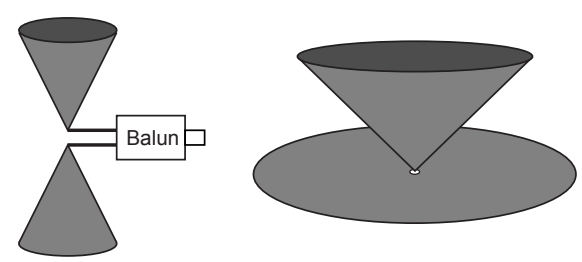

Fig. 4. Biconical and monopole antennas (not to scale).

for 3 and $8 \mathrm{GHz}$. These frequency dependent characteristics are well known in literature (Kraus, 1950; Schelkunoff, 1952).

These frequency dependent radiation patterns cannot generate a homogenous illumination of the whole room. Objects below the antenna ground plane will be hardly illuminated. To validate the whole room it is necessary to perform at least two measurements in each polarization plane (antenna up/down and left/right). Thus four measurements have to be performed. Nevertheless the illumination is very inhomogeneous. The cable routing by itself is a real problem in this case. A solution would be the use of smaller cones and especially smaller metallic counterpoises for higher frequencies. But this is not practicable.

However biconical antennas exist, which provide an excellent dipole type antenna pattern in the frequency range up to $3 \mathrm{GHz}$. Excited by this, a German company developed a small biconical antenna with a dipole type pattern for 3$18 \mathrm{GHz}$. The $3 \mathrm{~dB}$ beam-width decreases with increasing frequency. This is visible in the radiation pattern in Fig. 7 , which is based on a numerical simulation of the biconical antenna. But the antenna still provides a dipole radiation pattern. Therefore it is possible to perform the site validation with only two measurements (vertical and horizontal). In contrast to the monopole antenna a better illumination of the room is possible. 


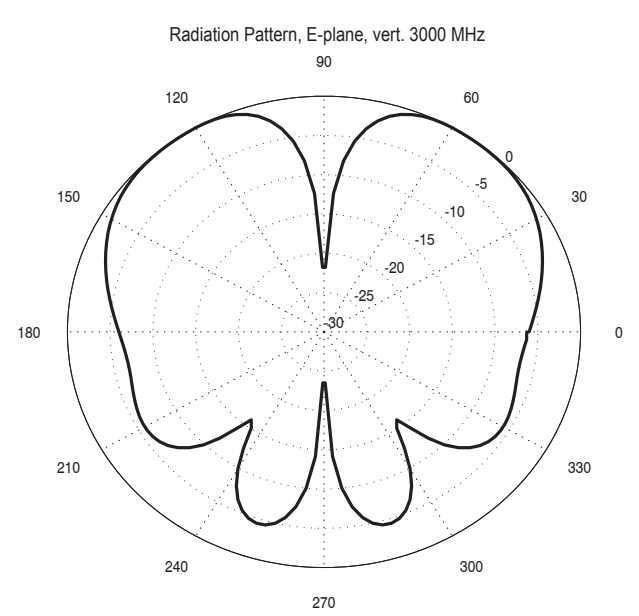

Fig. 5. Radiation pattern Monopole $3 \mathrm{GHz}$.

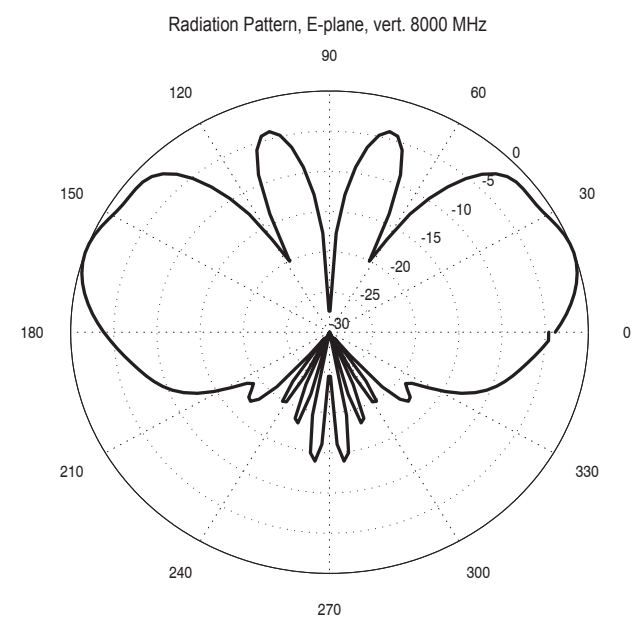

Fig. 6. Radiation pattern Monopole $8 \mathrm{GHz}$.

\section{Validation procedure}

\subsection{Basic idea}

Several kinds of validations have been discussed in the different CDs (CISPR, 2004). The site validation method tests a specified cylindrical test volume, typically located above the center of the turntable, for the combination of site, receive antenna and absorbing material placed on the ground plane (if needed to meet the criterion). The test volume has to enclose the EUT including cabling. The Site-VSWR method measures the standing wave along a line in the room - caused by unwanted reflections and resulting interferences. This is comparable to the one-dimensional standing wave on a transmission line, which occurs if the line is not terminated with its characteristic impedance. The VSWR is calculated from the maximum and minimum voltage of the standing wave on the line. The Site-VSWR method uses the measured maximum and minimum electric field strength. It is necessary to keep in mind, that in reality a three dimensional standing

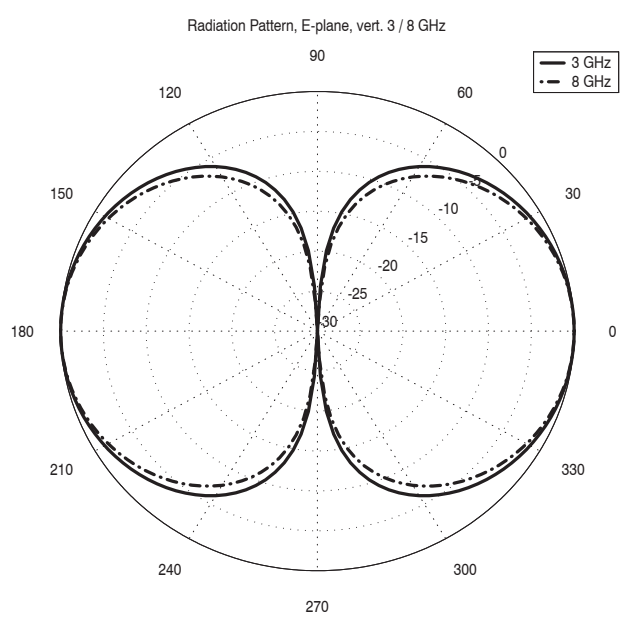

Fig. 7. Radiation pattern of biconical antenna at $3 \mathrm{GHz}$ and $8 \mathrm{GHz}$.

wave exists in the room but the Site-VSWR method measures only a small part of it.

The transmit antenna (biconical antenna) is located at specified positions in the test volume (see Fig. 8). The receive antenna for the validation has to be the same antenna, which will be used for measurements later on. Due to the larger beam-width a stacked log.-per--antenna is recommended.

Furthermore the reciprocal method is possible by moving a field probe in the test volume as receive antenna. In this case the normal receive antenna for EUT measurements is the transmitting antenna. It is allowed to change the transmitting and receiving antenna, because the system is passive and linear (reciprocity theorem).

For each frequency the maxima and minima of all measured voltages at the defined positions have to be determined and the Site-VSWR will be calculated with the following equation:

$s_{V S W R}=\max \left\{V_{\text {meas. } .}[d B]\right\}-\min \left\{V_{\text {meas. } .}[d B]\right\}$

In a free-space test environment, the Site-VSWR is directly related to the influence of undesired reflections (AppelHansen, 1973). The FAR or semi-anechoic chamber (SAC) fulfils the standard if the $s_{V S W R}$ is below a limit of about $3.5 \mathrm{~dB}$. This site acceptance criterion was developed by considering the desired magnitude of site effects, combined with influence quantities due to the instrumentation and procedures used to perform the site validation. Table 1 lists the parameters and their values used to establish the $3.5 \mathrm{~dB}$ criterion.

\subsection{New Draft (CD December 2004)}

In the new Draft (December 2004) the arrangement of measurement positions has been changed. As depicted in Figs. 8 and 9 at each position six measurements have to be done at non-equidistant positions. For the front position the distances $\Delta s$ relative to position $F 6$ are $2 \mathrm{~cm}, 10 \mathrm{~cm}, 18 \mathrm{~cm}, 30 \mathrm{~cm}$ and 
Table 1. Uncertainty budget of the site-VSWR validation method and resulting acceptance criteria.

\begin{tabular}{lll}
\hline Influence & Probability Distribution & Value $(\mathrm{k}=1) / \mathrm{dB}$ \\
\hline Site Imperfections (Target) & Normal & \pm 1.0 \\
Repeatability of $s_{V S W R}$ validation method & Normal & \pm 1.0 \\
Source Non-Ideal Characteristics & Rectangular & \pm 1.0 \\
(Pattern Deviations, Balance, Cross Polarization) & & \\
\hline Standard uncertainty & Sum of influence Quantities & \pm 1.65 \\
& $($ RSS combined) & \\
Expanded uncertainty $k=2$ & $=\left[1^{2}+1^{2}+(1.5 / 1.73)^{2}\right]^{0.5}$ & \\
& $S_{V S W R}$ tolerance value & $\mathrm{s}_{V S W} \leq 3.5$ \\
& $=V \max _{\mathrm{dB}}-V \min _{\mathrm{dB}}$ & \\
\hline
\end{tabular}
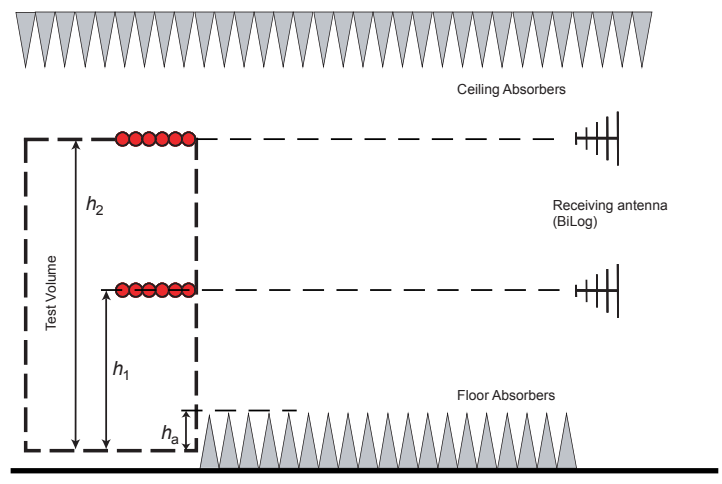

Fig. 8. Vertical section of the validated volume.

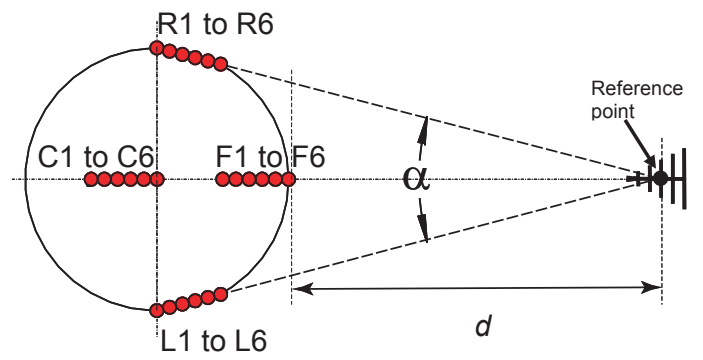

Fig. 9. Horizontal section at $h_{1}$ of the validated volume.

$40 \mathrm{~cm}$. The front positions in height $h_{1}$ and $h_{2}$ are mandatory. For floor standing equipment that cannot be raised, a height $h_{a}$ of $30 \mathrm{~cm}$ may be obstructed by absorbers placed on the ground of the SAC. The other positions depend on the receive antenna half power beam-width, absorber placement (for SAC) and dimensions of the test volume. The necessary points are defined in a given flow-chart in the standard.

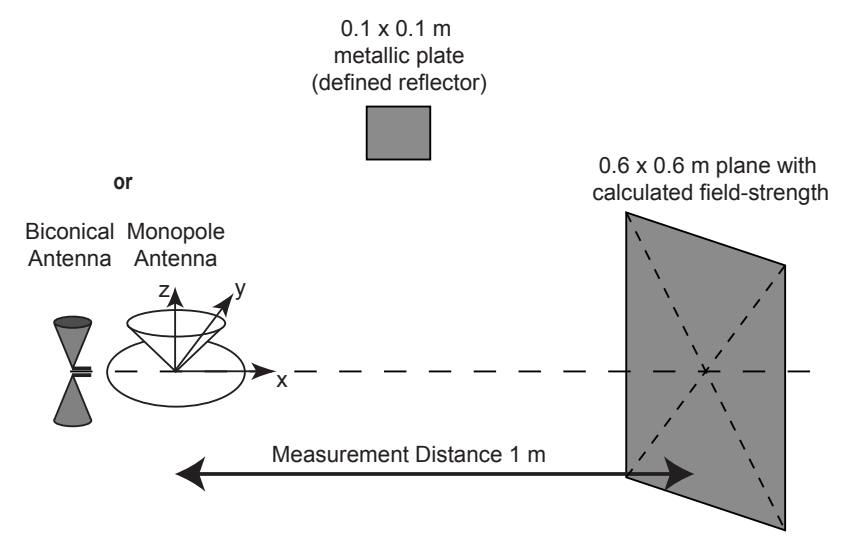

Fig. 10. Schematic of the Model.

\section{Calculations and measurements}

\subsection{Description of the model}

A numerical model has been developed to get a better insight into the transmit antenna pattern influence. It consists of only one antenna (monopole or biconical) placed at the origin (Fig. 10). The program CONCEPT has been used for the calculations, which is based on the Method of Moments. The antenna is fed with $1 \mathrm{~V}$ at $50 \mathrm{Ohms}$. The field has been calculated in the y-z-plane with the center position $(1 \mathrm{~m}, 0 \mathrm{~m}, 0 \mathrm{~m})$ and the dimension $(60 \times 60 \mathrm{~cm})$. The metallic reflector is $10 \times 10 \mathrm{~cm}$ and it is located in the $\mathrm{z}$-x-plane at $(0.5 \mathrm{~m}, 1 \mathrm{~m}, 0 \mathrm{~m})$. The center position of the plate is moved in z-direction only.

At each frequency a different interference pattern exists and the calculations have been carried out exemplarily for the frequency of $9 \mathrm{GHz}$. Thus it is possible to visualize the field pattern for the monopole and biconical antenna for special positions of the reflector. During the validation procedure (Site-VSWR) the interference pattern would be validated for all frequencies. 


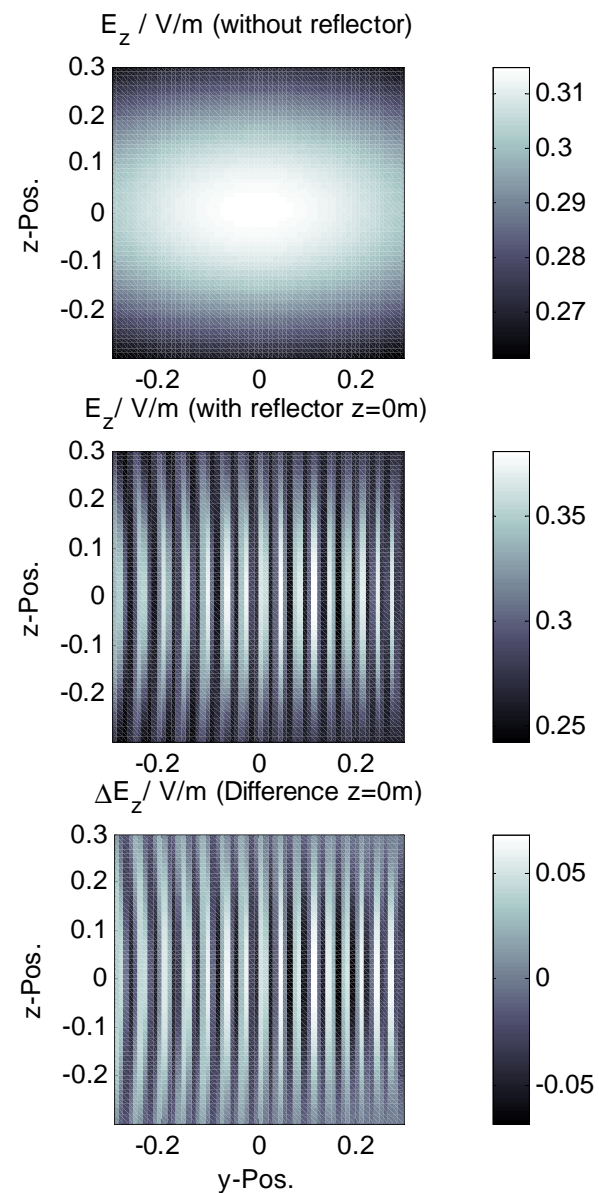

Fig. 11. Biconical $-E_{z}$ meas. plane (refl. at $\mathrm{z}=0$ ).

\subsection{Calculated field distribution}

\subsubsection{Metallic reflector at $\mathrm{z}=0 \mathrm{~m}$}

Figure 11 shows the z-component of the electrical field strength in the measurement plane caused by the biconical antenna. The first diagram shows the field without the reflector. The maximum of the electric field strength is in the middle of the plane due to the dipole radiation pattern. The diagram in the middle depicts the field with the reflector. The interference pattern is quite obvious. The last figure shows the difference of both field strengths.

Figure 12 depicts the z-component of the electrical field strength in the measurement plane with the monopole antenna as radiator. The maximum is not in the center, as the antenna has a main lobe with about $23^{\circ}$.

\subsubsection{Moved metallic reflector}

In Fig. 13 the field distribution with the reflector plate at $\mathrm{z}=-$ $40 \mathrm{~cm}$ (below the $\mathrm{x}-\mathrm{y}$-plane at $\mathrm{z}=0$ ) is depicted for the biconical antenna. The interference pattern changes significantly and the minima and maxima values in the difference pattern decrease from $\pm 0.05 \mathrm{~V}$ to $\pm 0.01 \mathrm{~V}$. This is understandable,

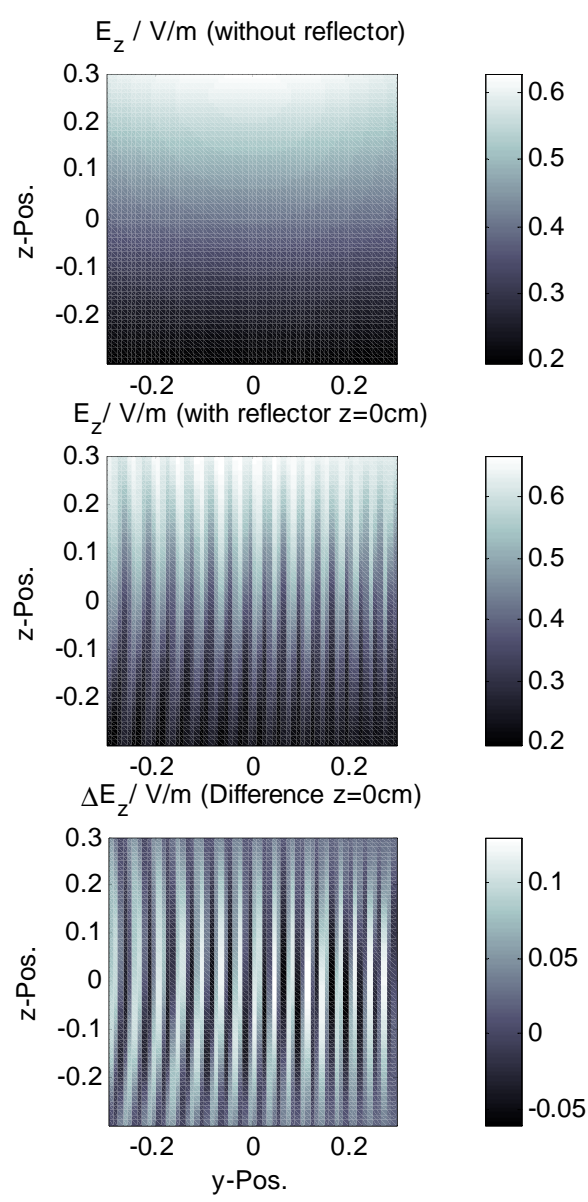

Fig. 12. Monopole $-E_{z}$ meas. plane (refl. at $\mathrm{z}=0$ ).

as the distance between the direct signal path and the metal plate is getting larger and the optical reflection (ray optics) is not fulfilled contrary to the condition in Fig. 10.

Figure 14 shows the field distribution with the reflector plate at $\mathrm{z}=-40 \mathrm{~cm}$ for the monopole antenna. The interference pattern is different from the pattern produced by the biconical antenna. But the Site-VSWR is based on the measurement of the interference pattern, which is caused by reflections. Therefore the radiation pattern of the transmit antenna has a significant influence on the Site-VSWR with identical reflectors.

Theoretically the influence on the Site-VSWR must be identical if the reflecting plate is $40 \mathrm{~cm}$ above or below the $x-y-p l a n e$ at $z=0$. The influence of the reflector on the measurement in a FAR is the same in both positions. Numerical calculations show, that the interference pattern of the biconical antenna is identical for both positions - it is just mirrored. This results from the symmetrical antenna pattern of the biconical antenna. The interference patterns of the monopole antenna for both positions of the reflector whereas are not identical. The reason for this is the multi-lobe antenna pattern of the monopole antenna. 

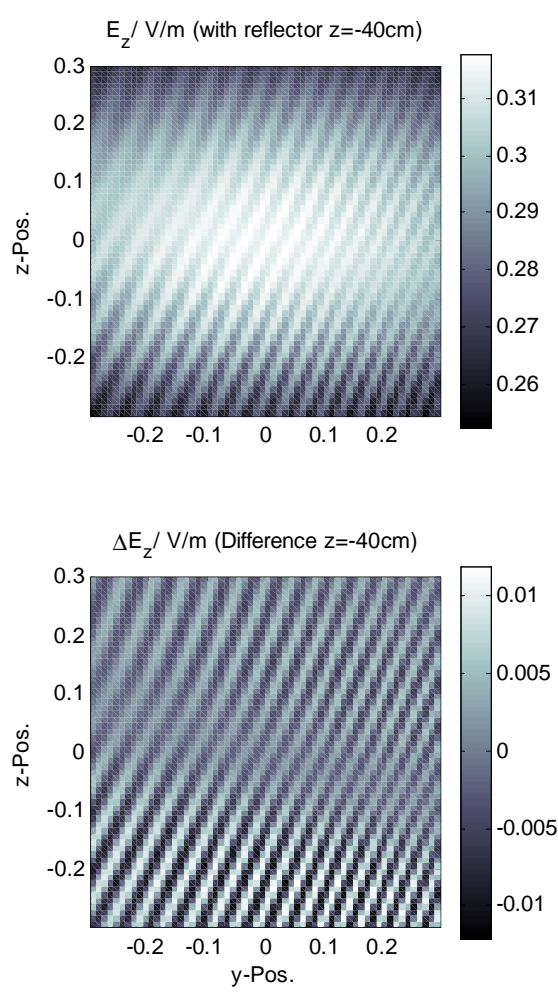

Fig. 13. Bicon. $-E_{z}$ meas. plane (refl. at $\left.\mathrm{z}=-40 \mathrm{~cm}\right)$.

\subsubsection{Receiving antenna influence}

The numerical calculations are independent of the receive antenna. But the validation procedure has to be carried out with the receive antenna which will be also used for the measurements. Further measurements showed that a high directional receiving antenna neglects reflections, as the $3 \mathrm{~dB}$ beamwidth is too small. The smaller the beam width - the lower the Site-VSWR. That is why the receive antenna characteristics have to be defined for the validation procedure. Further problems with antennas above $1 \mathrm{GHz}$, which have to be considered, are elucidated in (Windler and Camell, 2001).

\subsection{Measurements of the Site-VSWR}

The following diagrams show measurements of the SiteVSWR in a $3 \mathrm{~m}$ FAR with both antennas. The abovedepicted effects with the frequency dependent radiation pattern are quite obvious with the monopole antenna in Fig. 15 (right). The monopole overestimates directions at special frequencies, due to its radiation characteristics.

\section{Conclusion}

The measurements show, that the Site-VSWR method is suitable for FAR validation above $1 \mathrm{GHz}$. Nevertheless every test site has a certain inaccuracy. These values of the inaccuracy are taken into consideration and also defined in the
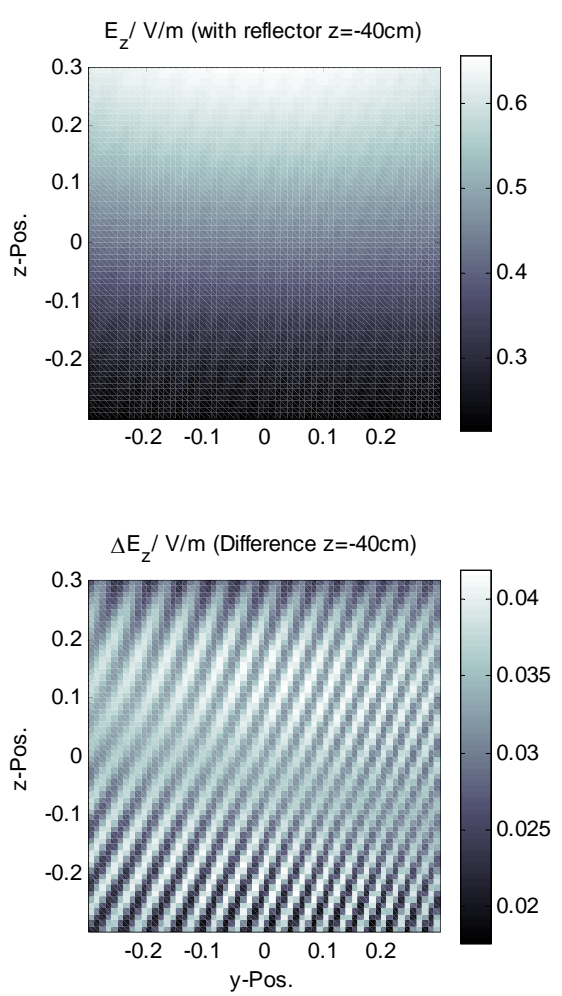

Fig. 14. Monop. $-E_{z}$ meas. plane (refl. at $\mathrm{z}=-40 \mathrm{~cm}$ ).

coming standard. To obtain comparable results between different FARs the transmit and receive antenna characteristics have to be taken into account during the validation. Without considering the antenna pattern it would be possible to use antennas with high directivity and the FAR will pass during the validation. But during measurements there will be problems due to possible reflections caused by the radiation pattern of the EUT. Therefore the standardization is concerned in defining the necessary antenna pattern especially for the transmit antenna.

The transmit antenna needs a dipole type radiation pattern. With vertical and horizontal polarization it is possible to illuminate the whole room. The monopole antenna with its frequency dependent multi-lobe radiation pattern is not suited for the site validation. The biconical antenna seems to be suited, due to its dipole type radiation pattern and broadband characteristics.

\section{References}

Appel-Hansen, J.: Reflectivity Level of Radio Anechoic Chambers, IEEE Trans. Antennas and Propagation, Vol. AP-21, No. 4, 490 498, July 1973.

CENELEC Report R210-010: Electromagnetic CompatibilityEmission measurements in Fully Anechoic Chambers, European Committee for Electrotechnical Standardization, Brussels, June 2002.

CISPR A/531/CD: Site Evaluation above $1 \mathrm{GHz}$, Committee International Special des Perturbation Radioelectrique, 2004. 

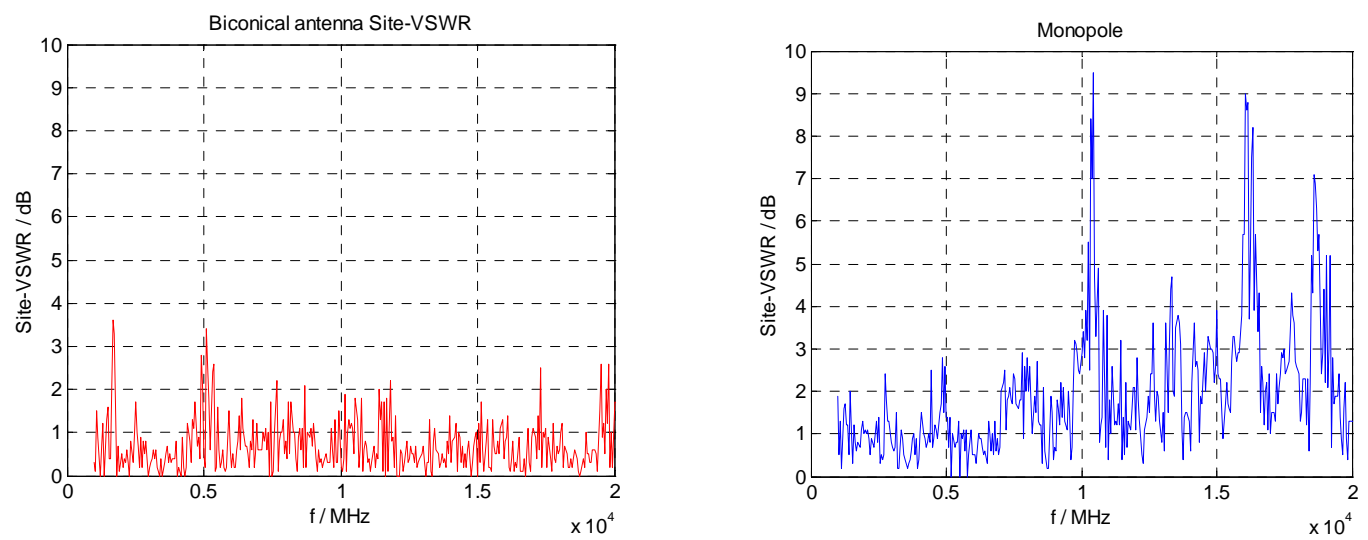

Fig. 15. Site-VSWR with biconical antenna and with monopole antenna.

DIN EN 50147-2:1996-07: Anechoic chambers - Part 2: Alternative test site suitability with respect to site attenuation, 1996.

Holloway, C., DeLyser, R , German, R., McKenna, P., and Kanda, M.: Comparison of Electromagnetic Absorber Used in Anechoic and Semi-Anechoic Chamber for Emission and Immunity Testing of Digital Devices, IEEE Trans. EMC, Vol. 39, No. 1, 33-47, Feb. 1997.

IEEE Standard Test Procedures for Antennas, ANSI/IEEE Std 1491979.
Kraus, J. D.: Antennas, McGraw-Hill, 1950.

Schelkunoff, S. A.: Advanced Antenna Theory, New York: Wiley, 1952.

Windler, M. J. and Camell, D.: Research on site qualification above $1 \mathrm{GHz}$, Proc. 15th Int. Zurich Symp. on electromagnetic Compatibility, Switzerland, 333-336, Feb. 18-20, 2003.

Windler, M. J. and Camell, D.: Measuring Antennas above $1 \mathrm{GHz}$, Proc. 14th International Zurich Symp. on Electromagnetic Compatibility (Supplement), Switzerland, 91-95, 2001. 